\title{
The change in high-sensitivity troponin-T as a risk factor for significant coronary stenosis in patients with acute coronary syndrome
}

Min Chul Kim, Seok Oh ${ }^{\star}$, Youngkeun Ahn, Keumyi Moon, Joon Ho Ahn, Dae Young Hyun, Kyung Hoon Cho, Doo Sun Sim, Young Joon Hong, Ju Han Kim, Myung Ho Jeong, Jeong Gwan Cho, and Jong Chun Park

Division of Cardiology, Department of Internal Medicine, Chonnam National University Hospital, Chonnam National University Medical School, Gwangju, Korea

Received: March 9, 2020

Revised : April 12, 2020

Accepted: April 26, 2020

\section{Correspondence to}

Youngkeun Ahn, M.D.

Division of Cardiology,

Department of Internal

Medicine, Chonnam National

University Hospital, Chonnam

National University Medical

School, 42 Jebongro,

Dong-gu, Gwangju 61469,

Korea

Tel: +82-62-220-4764

Fax: +82-62-224-4764

E-mail: cecilyk@hanmail.net https://orcid.org/0000-00032022-9366

*These authors contributed equally to this work.
Background/Aims: High-sensitivity cardiac troponin (hs-TnT) assays detect very low levels of cardiac troponin. This study examined the interval change between initial and subsequent hs-TnT levels and evaluated its ability to predict significant coronary stenosis.

Methods: The study analyzed 163 patients who presented with acute coronary syndrome (ACS) and underwent coronary angiography (CAG) between April 2014 and May 2018. The o and 3-hour hs-TnT were checked. The patients were subdivided into positive $(n=32)$ and negative $(n=131)$ interval change groups. The presence of significant coronary artery stenosis on CAG in the two groups was compared.

Results: The positive interval change group was older and had higher $\mathrm{o}$ and 3-hour hs-TnT and blood glucose levels than the negative interval change group. Significant coronary stenosis was more common in the positive interval change group than in the negative interval change group (68.8\% vs. $23.7 \%, p=0.001$ ). However, vasospasm was more common in the negative interval change group (6.3\% vs. $31.3 \%, p=0.003)$. The positive interval change group had higher rates of bifurcation lesions and received more percutaneous coronary intervention. In multivariate analysis, age, interval change of serial hs-TnT and diabetes mellitus were independent predictors of significant coronary artery stenosis.

Conclusions: This study identified a relationship between the serial change in cardiac biomarkers and the presence of significant coronary stenosis in patients with ACS. Serial hs-TnT change was associated with real angiographic stenosis in patients with ACS.

Keywords: Acute coronary syndrome; Troponin T; Coronary stenosis

\section{INTRODUCTION}

In South Korea, the incidence of acute coronary syndrome (ACS), including acute myocardial infarction (AMI), is increasing gradually and becoming a serious socioeconomic problem [1]. In the emergency setting, an early diagnosis of myocardial infarction (MI) is advantageous for patients and the physicians treating them [2]. Therefore, several rapid diagnosis methods have been devised, and cardiac biomarkers are considered the most important [3-7]. Cardiac troponin is the biomarker of choice for diagnosing myocardial necrosis, as it is 
considered the most sensitive and specific biomarker of myocardial injury [8,9]. High-sensitivity troponin (hsTn) has emerged for the assessment of acute chest pain and the improved sensitivity allows the identification of practically any amount of myocardial necrosis $[10,11]$. The marked evolution of these cardiac biomarker assays has reduced the time interval to the second Tn assessment. If hs-Tn is available, a rapid 'rule-out' protocol (3-hour hs-Tn) is recommended [12-14]. Furthermore, Reichlin et al. [15] introduced a 1-hour 'rule-out' algorithm as a faster diagnostic tool than the 3-hour 'ruleout' protocol.

However, no studies have examined a rapid 'rule-out' algorithm using high-sensitivity troponin $\mathrm{T}$ (hs-TnT) in Korean patients with ACS. In addition, there is little information on the prediction of significant coronary artery stenosis using hs-TnT. We hypothesized that if the change in myocardial enzymes is significant, there would be a high likelihood that significant coronary artery stenosis is present in patients with chest pain and suspected non-ST-segment elevation ACS (NSTE-ACS).

\section{METHODS}

\section{Study population}

From April 2014 to May 2018, 484 patients presented to our tertiary center with acute chest pain suspected of being ACS, which developed within 6 hours. The initial hs-TnT (or o-hour hs-TnT) was tested routinely. To estimate the interval change in cardiac enzymes, hs-TnT was re-checked 3 hours after presentation and defined as the 3-hour hs-TnT. The study flowchart is shown in Fig. 1. The following patients were excluded from the study: (1) those with duration of chest pain > 6 hours; (2) those with possible 'rule-in' MI; (3) those with medical conditions that could affect Tn levels (e.g., systemic infection, acute cerebrovascular accident, tachyarrhythmia, renal disease, heart failure, or other acute medical conditions rather than ACS); and (4) those who previously underwent an interventional cardiac procedure. Patients with a o-hour hs-TnT $\geq 0.1 \mathrm{ng} / \mathrm{mL}$ or who presented with electrocardiogram findings suggesting ST-segment elevation were excluded because they were identified as a 'rule-in' MI. Patients with a history of coronary artery disease, previous percutaneous coronary intervention

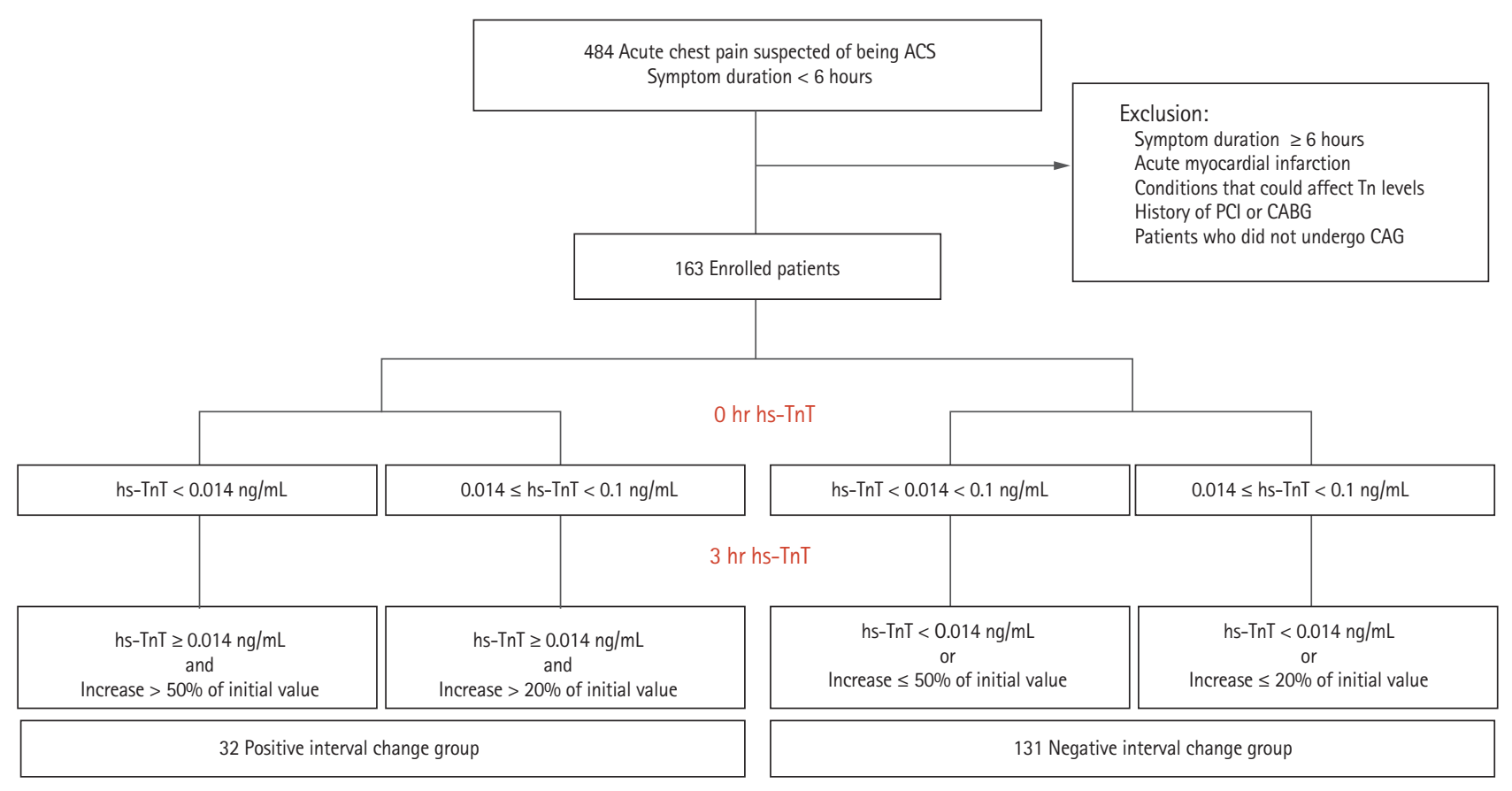

Figure 1. Study flowchart. ACS, acute coronary syndrome; CABG, coronary artery bypass graft; CAG, coronary angiography; hs-TnT, high-sensitivity troponin T; PCI, percutaneous coronary intervention. 
(PCI), or previous coronary artery bypass graft were also excluded. The patients who required cardiopulmonary resuscitation during admission, were in cardiogenic shock, or had chronic heart failure were excluded because these conditions may affect the initial Tn levels. Patients who did not undergo coronary angiography (CAG) were excluded. Ultimately, the study enrolled 163 patients. These were subdivided into hs-cTnT positive $(\mathrm{n}=32)$ and negative $(\mathrm{n}=131)$ interval change groups. This study was a prospective, non-randomized, observational study. The study protocol was approved by the Chonnam National University Hospital Institutional Review Board (IRB No. CNUH-2014-124). Informed consent was obtained from all study participants.

\section{Study definitions and endpoint}

Blood samples were collected in serum-separating tubes. Samples were obtained from 484 patients immediately after emergency room visits. The hs-TnT level was measured by Roche e411 (Roche Diagnostics, Rotkreuz, Switzerland). An interval change in serial hs-TnT was defined as follows: (1) 3-hour hs-TnT $\geq 0.014 \mathrm{ng} / \mathrm{mL}$ and more than $50 \%$ greater than o-hour hs-TnT in patients with a o-hour hs-TnT < $0.014 \mathrm{ng} / \mathrm{mL}$; or (2) 3-hour hs-TnT $\geq 0.014 \mathrm{ng} / \mathrm{mL}$ and more than $20 \%$ greater than o-hour hs-TnT in patients with a o-hour hs-TnT $\geq 0.014 \mathrm{ng} /$ $\mathrm{mL}$. The current cutoff value for the hs-TnT assay is $0.014 \mathrm{ng} / \mathrm{mL}$ [9]. Although the 2015 European Society of Cardiology (ESC) guidelines recommend invasive management for patients with acute chest pain if 3-hour hsTnT is greater than $0.014 \mathrm{ng} / \mathrm{mL}$ [5], we defined positive or negative interval change groups according to an older guideline that subdivided patients with 3-hour hs-TnT $\geq 0.014 \mathrm{ng} / \mathrm{mL}$ into two groups [16]. The clinical manifestations of NSTE-ACS reference the 2015 ESC guidelines, as follows: (1) prolonged (over 20 minutes) angina at rest; (2) new-onset class II or III angina of the Canadian Cardiovascular Society (CCS) classification; (3) recent destabilization of previously stable angina with at least CCS class III angina (crescendo angina); and (4) post-MI angina. The term non-ST-segment elevation myocardial infarction (NSTEMI) was used when there was evidence of myocardial necrosis (defined as an elevated troponin value with at least one value above the 99th percentile upper reference limit) and the aforementioned clinical features [5]. In this study, hs-TnT was used as a cardiac biomarker to determine whether the subjects had unstable angina or MI. In this study, significant coronary artery stenosis was confirmed by CAG and defined as follows: ( 1 ) $\geq 50 \%$ diameter stenosis identified in the left main coronary artery at CAG; or (2) $\geq 70 \%$ diameter stenosis identified in coronary arteries other than the left main coronary artery at CAG. Multi-vessel disease was determined when there were significant coronary stenoses in two or more epicardial coronary arteries. A bifurcation lesion was defined as narrowing of a coronary artery adjacent to or involving the origin of a significant side branch [17]. Vasospasm was defined as a > 90\% reduction in diameter on the ergonovine provocation test or a spontaneous, dynamic $>90 \%$ decrease in diameter observed during CAG.

The medical and social histories were obtained using a questionnaire. The conventional cardiac risk factors included were hypertension, diabetes, dyslipidemia, stroke, and smoking status. Body data such as height $(\mathrm{m})$ and weight $(\mathrm{kg})$ were measured by experienced nurses and body mass index (BMI, $\mathrm{kg} / \mathrm{m}^{2}$ ) was calculated. Blood was tested in all subjects on admission, including white blood cell count, hemoglobin level, platelet count, serum glucose, serum creatinine, and hs-TnT. The hsTnT was measured at presentation and 3 hours later. All study subjects underwent echocardiography and CAG. The left ventricular ejection fraction was considered an indicator of ventricular systolic function on transthoracic echocardiography. Patients who had an interval change underwent CAG between admission and hospital discharge, while patients who were negative for an interval change underwent CAG within 1 to 2 months of admission via a hospital visit. The coronary arteries were assessed quantitatively and qualitatively using CAG. If significant coronary artery stenosis was found at CAG, PCI was performed simultaneously.

\section{Statistical analysis}

All statistical analyzes were performed using SPSS for Windows version 25.0 (IBM Co., Armonk, NY, USA). Continuous variables are expressed as the mean \pm standard deviation. Discrete (categorical) variables are expressed as percentages and counts. Student's $t$ test was used to analyze the difference between two independent groups of continuous variables. In the analysis of categorical variables, the difference between the observed 
Table 1. Baseline characteristics

\begin{tabular}{|c|c|c|c|}
\hline Characteristic & $\begin{array}{l}\text { Positive interval change } \\
\qquad(\mathrm{n}=32)\end{array}$ & $\begin{array}{l}\text { Negative interval change } \\
\qquad(\mathrm{n}=131)\end{array}$ & $p$ value \\
\hline Age, yr & $60.09 \pm 10.40$ & $57.79 \pm 11.74$ & 0.312 \\
\hline Age $\geq 65 \mathrm{yr}$ & $11(34.4)$ & $37(28.2)$ & 0.495 \\
\hline Male sex & $23(71.9)$ & $86(65.6)$ & 0.502 \\
\hline $\mathrm{BMI}, \mathrm{kg} / \mathrm{m}^{2}$ & $25.22 \pm 2.87$ & $24.53 \pm 2.97$ & 0.268 \\
\hline $\mathrm{BMI} \geq 25 \mathrm{~kg} / \mathrm{m}^{2}$ & $16(55.2)$ & $41(36.9)$ & 0.075 \\
\hline o hr hs-TnT, ng/mL & $0.020 \pm 0.014$ & $0.011 \pm 0.008$ & 0.001 \\
\hline o hr hs-TnT $\geq 0.014$ ng/mL & $18(56.3)$ & $19(16.0)$ & $<0.001$ \\
\hline $3 \mathrm{hr}$ hs-TnT, ng/mL & $0.150 \pm 0.206$ & $0.010 \pm 0.007$ & 0.001 \\
\hline $3 \mathrm{hr}$ hs-TnT $\geq 0.014 \mathrm{ng} / \mathrm{mL}$ & $32(100)$ & $16(13.6)$ & $<0.001$ \\
\hline White blood cell, × $1,000 / \mu \mathrm{L}$ & $7,950 \pm 2140$ & $7,200 \pm 1,990$ & 0.070 \\
\hline Hemoglobin, g/dL & $14.15 \pm 1.52$ & $14.17 \pm 1.49$ & 0.971 \\
\hline Platelet, $\times 1,000 / \mu \mathrm{L}$ & $220.17 \pm 57.96$ & $226.56 \pm 57.28$ & 0.585 \\
\hline Glucose, mg/dL & $151.90 \pm 57.92$ & $115.07 \pm 29.68$ & 0.002 \\
\hline Creatinine, $\mathrm{mg} / \mathrm{dL}$ & $0.76 \pm 0.18$ & $0.77 \pm 0.18$ & 0.963 \\
\hline \multicolumn{4}{|l|}{ Previous medical history } \\
\hline Hypertension & $15(50.0)$ & $44(35 \cdot 5)$ & 0.142 \\
\hline Diabetes mellitus & $7(23 \cdot 3)$ & $18(14 \cdot 5)$ & 0.240 \\
\hline Dyslipidemia & $9(30.0)$ & $26(21.0)$ & 0.333 \\
\hline Stroke & o & $3(2.4)$ & 0.617 \\
\hline Current or ex-smoking & $13(43 \cdot 3)$ & $52(41.9)$ & 0.889 \\
\hline Left ventricular ejection fraction, \% & $65.13 \pm 7.81$ & $65.60 \pm 6.00$ & 0.759 \\
\hline
\end{tabular}

Values are presented as mean \pm SD or number (\%).

BMI, body mass index; hs-TnT, high-sensitivity troponin T.

and expected frequencies was confirmed by Pearson's chi-square test. To demonstrate the impact of the interval change in hs-TnT on the presence of significant coronary artery stenosis, the odds ratio (OR) and 95\% confidence interval(CI) were calculated using univariable and multivariable logistic regression analysis. The ORs were adjusted according to the variables that had statistical significance $(p<0.05)$ in the univariable analysis in the multivariable model.

\section{RESULTS}

\section{Baseline characteristics}

Table 1 compares the baseline clinical characteristics. There was no significant difference between the two groups in age $(60.09 \pm 10.40$ years vs. $57.79 \pm 11.74$ years, $p=0.312)$, proportion $\geq 65$ years old $(34.4 \%$ vs. $28.2 \%$, $p=0.495)$, or proportion of males (71.9\% vs. $65.6 \%$, $p=0.502$ ). The positive interval change group had higher o-hour hs-TnT level (0.020 $\pm 0.014 \mathrm{ng} / \mathrm{mL}$ vs. $0.011 \pm$ $0.008 \mathrm{ng} / \mathrm{mL}, p=0.001$ ), proportion of o-hour hs-TnT $\geq 0.014 \mathrm{ng} / \mathrm{mL}$ ( $56.3 \%$ vs. $16.0 \%, p=0.001$ ), and 3 -hour hsTnT level ( $0.150 \pm 0.206$ ng/mL vs. $0.010 \pm 0.007 \mathrm{ng} / \mathrm{mL}$, $p=0.050$ ) than the negative interval change group. The laboratory tests did not differ significantly, except for the serum glucose level $(151.90 \pm 57.92 \mathrm{ng} / \mathrm{mL}$ vs. $115.07 \pm$ $29.68 \mathrm{mg} / \mathrm{dL}, p=0.031$ ). There was no difference in conventional cardiac risk factors such as hypertension, diabetes mellitus, and dyslipidemia in both groups.

\section{Coronary angiographic characteristics}

Table 2 summarizes the angiographic findings of both groups. There was a marked difference in the presence 
Table 2. Angiographic and procedural characteristics

\begin{tabular}{|c|c|c|c|}
\hline Characteristic & $\begin{array}{l}\text { Positive interval change } \\
\qquad(\mathrm{n}=32)\end{array}$ & $\begin{array}{l}\text { Negative interval change } \\
\qquad(\mathrm{n}=131)\end{array}$ & $p$ value \\
\hline Significant coronary stenosis & $22(68.8)$ & $31(23.7)$ & 0.001 \\
\hline Pre-TIMI flow o-2 & $17(53.1)$ & $18(13.7)$ & $<0.001$ \\
\hline Multivessel disease & $6(18.8)$ & $11(8.4)$ & 0.086 \\
\hline Bifurcation lesion & $4(12.5)$ & $3(2.3)$ & 0.028 \\
\hline Left main disease & $2(6.3)$ & $4(3.1)$ & 0.335 \\
\hline Vasospasm & $2(6.3)$ & $41(31.3)$ & 0.003 \\
\hline PCI & $21(65.6)$ & $30(22.9)$ & $<0.001$ \\
\hline Total number of stents & $1.47 \pm 0.61$ & $1.66 \pm 1.01$ & 0.486 \\
\hline Stent diameter per lesion, $\mathrm{mm}$ & $3.18 \pm 0.52$ & $3.07 \pm 0.50$ & 0.481 \\
\hline Stent length per lesion, mm & $23.98 \pm 7.67$ & $23.52 \pm 7.95$ & 0.842 \\
\hline Drug-eluting stent & $18(94 \cdot 7)$ & $27(93.1)$ & 0.819 \\
\hline
\end{tabular}

Values are presented as number (\%) or mean \pm SD.

TIMI, thrombolysis in myocardial infarction; PCI, percutaneous coronary intervention.

Table 3. Univariable logistic regression analysis for significant coronary stenosis

\begin{tabular}{|c|c|c|c|}
\hline Characteristic & Unadjusted OR & $95 \%$ CI & $p$ value \\
\hline Age & 1.052 & $1.019-1.086$ & 0.002 \\
\hline Male sex & 1.592 & $0.772-3.283$ & 0.208 \\
\hline $\mathrm{BMI} \geq 25 \mathrm{~kg} / \mathrm{m}^{2}$ & 1.260 & $0.628-2.525$ & 0.515 \\
\hline o hr hs-TnT $\geq 0.014$ ng/mL & 3.015 & $1.403-6.477$ & 0.005 \\
\hline $3 \mathrm{hr}$ hs-TnT $\geq 0.014 \mathrm{ng} / \mathrm{mL}$ & $4 \cdot 550$ & $2.184-9.478$ & $<0.001$ \\
\hline Interval change of serial hs-Tn'T ${ }^{\mathrm{a}}$ & 7.097 & $3.036-16.590$ & $<0.001$ \\
\hline Serum glucose, mg/dL & 1.011 & $1.002-1.020$ & 0.017 \\
\hline Serum creatinine, $\mathrm{mg} / \mathrm{dL}$ & 4.697 & $0.709-31.116$ & 0.109 \\
\hline Hypertension & 1.820 & $0.915-3.621$ & 0.088 \\
\hline Diabetes mellitus & 4.029 & $1.656-9.803$ & 0.002 \\
\hline Dyslipidemia & 1.537 & $0.704-3.357$ & 0.281 \\
\hline Previous history of stroke & 1.041 & $0.092-11.75^{8}$ & 0.974 \\
\hline Current or ex-smoking & 0.987 & $0.499-1.955$ & 0.971 \\
\hline Left ventricular ejection fraction, \% & 0.947 & $0.897-1.001$ & 0.053 \\
\hline
\end{tabular}

OR, odds ratio; CI, confidence interval; BMI, body mass index; hs-TnT, high-sensitivity troponin T.

${ }^{\mathrm{a}}$ Definition is described on the Method section.

of significant coronary artery stenosis (68.8\% vs. $23.7 \%$, $p=0.001)$. There was a higher proportion of reduced initial thrombolysis in myocardial infarction (TIMI) flow (i.e., initial TIMI flow o, I, and II) in the positive interval change group ( $53.1 \%$ vs. $13.7 \%, p<0.001$ ). The positive interval change group had a higher rate of bifurcation lesions (12.5\% vs. $2.3 \%, p=0.028$ ), but a lower proportion of vasospastic components (6.3\% vs. $31.3 \%, p=0.003)$. A higher proportion of the positive interval change group underwent PCI (65.6\% vs. $22.9 \%, p<0.001)$.

\section{Independent predictors of significant coronary artery stenosis}

The univariable and multivariable analyses of predictors of significant coronary stenosis are described in Tables 3 and 4 , respectively. In the univariable analyses, age, 
Table 4. Independent predictors of significant coronary stenosis

\begin{tabular}{lccc}
\hline Variable & Adjusted OR & $95 \%$ CI & p value \\
\hline Age & 1.054 & $1.013-1.097$ & 0.024 \\
Interval change of serial hs-TnT & 10.196 & $3.571-29.111$ & 0.001 \\
Diabetes mellitus & 3.390 & $1.216-9.451$ & 0.013 \\
\hline
\end{tabular}

OR, odds ratio; CI, confidence interval; hs-TnT, high-sensitivity troponin T.

${ }^{\mathrm{a}}$ Definition is described on the Method section.

o-hour hs-TnT, 3-hour hs-TnT, interval change of serial hs-TnT, serum glucose level and history of diabetes mellitus were associated with significant coronary stenosis (Table 3). Multivariable analysis was performed on these significant variables, and age (adjusted OR, 1.054; 95\% CI, 1.013 to 1.097; $p=0.024$ ), interval change of serial hs-TnT (adjusted OR, 10.196; 95\% CI, 3.571 to 29.111; $p=0.001$ ), and history of diabetes mellitus (adjusted OR 3.390; $95 \%$ CI, 1.216 to $9.451 ; p=0.013$ ) were identified as independent predictors of the presence of significant coronary artery stenosis (Table 4).

\section{DISCUSSION}

This study examined the association between significant coronary stenosis and the interval change of serial cardiac biomarkers. A higher proportion of significant stenosis was observed in the positive interval change group. In the positive group, the o and 3-hour hs-TnT were higher than in the negative group. As there was a higher rate of significant coronary stenosis in the positive group, the proportion undergoing PCI was also higher in that group. Multivariable analysis of the risk factors for significant coronary stenosis demonstrated that the interval change in serial cardiac biomarkers was the most important predictor of severe artery coronary stenosis angiographically.

The advent of the hs-TnT assay has led to the development of a method for the rapid assessment of 'rule-out' and 'rule-in' AMI $[9,12,18,19]$. Many studies have examined hs-TnT worldwide. Recent guidelines mention the early 'rule-out' method for AMI. This method is based on the hs-TnT assay and sampling at $\mathrm{o}$ and 1 hour. This algorithm includes two conditions to rule out MI: (1) hs$\mathrm{Tn}$ is a continuous variable and the probability of MI increases with hs-Tn; and (2) early absolute changes in the level within 1 hour can be interpreted as surrogates of absolute changes over 3 or 6 hours and add incremental diagnostic value to the assessment of cardiac troponin at admission. This 'rule-out' algorithm should always be integrated with a detailed clinical assessment. It is also mandatory to evaluate the 12-lead electrocardiogram and repeat blood sampling including Tn for all cases of ongoing or recurrent angina.

We hypothesized that cardiac troponin, especially hs-TnT, can predict the presence of angiographic significant coronary stenosis. In many cases of MI, actual significant coronary stenosis may not be present [20-23]. CAG is needed to determine whether stenosis exists. All NSTE-ACS cases that have a rise or fall in Tn compatible with MI are classified as high-risk patients, and should undergo CAG within 24 hours, according to the 2015 ESC guidelines [5]. All STEMI cases should undergo primary PCI within 12 hours, according to the 2017 ESC guidelines [24]. Indeed, the greater the likelihood of having significant coronary stenosis is, the greater the need for early revascularization.

A few studies describe the relationship between cardiac biomarkers and significant coronary stenosis. von Jeinsen et al. [25] noted that cardiac troponin-I is useful for diagnosing significant obstructive coronary artery disease in the hemodynamically stable setting of suspected AMI with special electrocardiographic findings, such as bundle branch block or a wide QRS complex. Sanchis et al. [26] measured hs-TnT at the time of presentation and after 6 hours in patients presenting with non-STE acute chest pain and elevated hs-TnT, estimated the maximum hs-cTnT and interval change in hsTnT, and optimized the cut-off values. They noted that low maximum and low interval changes in hs-TnT were associated with angiographically proven no-stenosis.

In this study, we selected 3-hour hs-TnT as the reference value for a change in cardiac biomarkers. The 
2015 ESC guidelines recommend applying the o/3-hour 'rule-out' algorithm of NSTE-ACS using the hs-TnT assay [5]. In accordance with prior studies regarding the hs-TnT assay, we set the upper normal limit of 3-hour hs-c'TnT as $0.014 \mathrm{ng} / \mathrm{mL}[9,27]$. We included NSTEMI with hs-TnT of at least $0.14 \mathrm{ng} / \mathrm{mL}$ and at most $0.1 \mathrm{ng} /$ $\mathrm{mL}$. In such patients, it may be difficult to predict the presence of significant coronary stenosis, so these may be considered 'gray zone' patients clinically. In these 'gray zone' cases, we found that the interval change in hs-TnT predicts some coronary stenotic lesions before CAG. This supports the evidence for performing CAG. As expected, both o and 3-hour hs-TnT were significantly higher in the positive interval change group. Interestingly, the proportion of patients with a significant vasospasm was statistically higher in the negative group. In other words, if ACS is suspected, but the serial changes in cardiac markers are not significant, vasospastic angina may contribute to the clinical situation. Vasospastic angina was diagnosed when spontaneous spasm or ergonovine-induced spasm occurred. If no spontaneous spasm occurred, the ergonovine provocation test was only performed in patients with clinical suspicion of vasospastic angina. However, the prevalence of vasospasm in the negative hs-TnT group was relatively high (6.3\% vs. 31.3\%, $p=0.003$ ). Therefore, routine vasospasm provocation test can be considered in patients with moderately elevated hs-TnT. The 1-hour 'rule-in' algorithm was introduced in the 2015 ESC guidelines. However, it was not available in our institution at the time of this study.

Our study had several limitations. First, there is no expert consensus on how to determine the interval change in cardiac biomarkers. Many studies define the interval change as the arithmetic difference between the o-hour cardiac o and subsequent estimated Tn level [12-14]. However, we determined the interval change using a slightly different method. Second, the symptom duration might have affected study outcomes. Patients with long symptom duration likely had pre-conditioned myocardium and rich collateral flows in coronary artery circulation, and these aspects might have influenced the study results. Although patients in the current study had symptom durations of less than 6 hours, there was no exact information regarding symptom duration in the case report form. Third, there was some diversity among the underlying clinical characteristics. Fourth, the study was conducted in a single medical center with a small sample size. Therefore, caution is needed when interpreting the results. Finally, this study did not include follow-up information on the clinical prognosis of the subjects. Therefore, large randomized trials should examine the clinical outcomes in both groups.

In conclusion, the change in serial hs-TnT was associated with real angiographic severe stenosis in patients with ACS. A multicenter study with a large number of patient samples is needed.

\section{KEY MESSAGE}

1. A higher proportion of significant stenosis was observed in the positive interval change group, according to the 3-hour algorithm (68.8\% vs. $23.7 \%)$.

2. Coronary vasospasm was more common in the negative interval change group (6.3\% vs. 31.3\%).

3. Serial high-sensitivity troponin $\mathrm{T}$ change was associated with real angiographic stenosis in patients with acute coronary syndrome.

\section{Conflict of interest}

No potential conflict of interest relevant to this article was reported

\section{Acknowledgments}

This study was supported by a grant of the Bio \& Medical Technology Development Program of the NRF funded by the Korean government, MSIP (2017M3A9E8023020), and National Research Foundation of Korea (2019R1A2C3003547).

\section{REFERENCES}

1. Statistics Korea. Deaths and death rates by cause (236 item), sex, and age (by five-year age group) [Internet]. Daejeon (KR): Statistics Korea, 2019 [cited 2020 Nov 5]. Available from: http://kostat.go.kr/portal/korea/kor_nw/1/6/2/index.board.

2. Kavsak PA, Worster A. For a rapid diagnosis of acute myocardial infarction, a sensitive troponin assay is needed in the near-patient testing setting. Expert Rev Cardiovasc 
Ther 2012;10:309-312.

3. Zhelev Z, Hyde C, Youngman E, et al. Diagnostic accuracy of single baseline measurement of elecsys troponin $\mathrm{T}$ highsensitive assay for diagnosis of acute myocardial infarction in emergency department: systematic review and metaanalysis. BMJ 2015;350:h15.

4. Rubini Gimenez M, Twerenbold R, Jaeger C, et al. One-hour rule-in and rule-out of acute myocardial infarction using high-sensitivity cardiac troponin I. Am J Med 2015;128:861870.

5. Roffi M, Patrono C, Collet JP, et al. 2015 ESC guidelines for the management of acute coronary syndromes in patients presenting without persistent ST-segment elevation: task force for the management of acute coronary syndromes in patients presenting without persistent ST-segment elevation of the European Society of Cardiology (ESC). Eur Heart J 2016;37:267-315.

6. Body R, Carley S, McDowell G, et al. Rapid exclusion of acute myocardial infarction in patients with undetectable troponin using a high-sensitivity assay. J Am Coll Cardiol 2011;58:1332-1339.

7. Bandstein N, Ljung R, Johansson M, Holzmann MJ. Undetectable high-sensitivity cardiac troponin $\mathrm{T}$ level in the emergency department and risk of myocardial infarction. J Am Coll Cardiol 2014;63:2569-2578.

8. Thygesen K, Mair J, Katus H, et al. Recommendations for the use of cardiac troponin measurement in acute cardiac care. Eur Heart J 2010;31:2197-2204.

9. Thygesen K, Mair J, Giannitsis E, et al. How to use highsensitivity cardiac troponins in acute cardiac care. Eur Heart J 2012;33:2252-2257.

10. Sanchis J, Bardaji A, Bosch X, et al. Usefulness of highsensitivity troponin $\mathrm{T}$ for the evaluation of patients with acute chest pain and no or minimal myocardial damage. Am Heart J 2012;164:194-200.

11. Thygesen K, Alpert JS, Jaffe AS, et al. Third universal definition of myocardial infarction. Eur Heart J 2012;33:25512567.

12. Keller T, Zeller T, Ojeda F, et al. Serial changes in highly sensitive troponin I assay and early diagnosis of myocardial infarction. JAMA 2011;306:2684-2693.

13. Reichlin T, Hochholzer W, Bassetti S, et al. Early diagnosis of myocardial infarction with sensitive cardiac troponin assays. N Engl J Med 2009;361:858-867.

14. Biener M, Mueller M, Vafaie M, et al. Comparison of a 3-hour versus a 6-hour sampling-protocol using high- sensitivity cardiac troponin $\mathrm{T}$ for rule-out and rule-in of non-STEMI in an unselected emergency department population. Int J Cardiol 2013;167:1134-1140.

15. Reichlin T, Schindler C, Drexler B, et al. One-hour ruleout and rule-in of acute myocardial infarction using high-sensitivity cardiac troponin T. Arch Intern Med 2012;172:1211-1218.

16. Hamm CW, Bassand JP, Agewall S, et al. ESC guidelines for the management of acute coronary syndromes in patients presenting without persistent ST-segment elevation: the task force for the management of acute coronary syndromes (ACS) in patients presenting without persistent ST-segment elevation of the European Society of Cardiology (ESC). Eur Heart J 2011;32:2999-3054.

17. Louvard Y, Thomas M, Dzavik V, et al. Classification of coronary artery bifurcation lesions and treatments: time for a consensus! Catheter Cardiovasc Interv 2008;71:175-183.

18. Reichlin T, Twerenbold R, Reiter M, et al. Introduction of high-sensitivity troponin assays: impact on myocardial infarction incidence and prognosis. Am J Med 2012;125:12051213.

19. Haaf P, Drexler B, Reichlin T, et al. High-sensitivity cardiac troponin in the distinction of acute myocardial infarction from acute cardiac noncoronary artery disease. Circulation 2012;126:31-40.

20. Virmani R, Kolodgie FD, Burke AP, Farb A, Schwartz SM. Lessons from sudden coronary death: a comprehensive morphological classification scheme for atherosclerotic lesions. Arterioscler Thromb Vasc Biol 2000;20:1262-1275.

21. Mann JM, Davies MJ. Vulnerable plaque: relation of characteristics to degree of stenosis in human coronary arteries. Circulation 1996;94:928-931.

22. Frycz-Kurek AM, Gierlotka M, Gasior M, et al. Patients with no significant lesions in coronary arteries and ST-segment elevation myocardial infarction have worse outcome than patients with non-ST-segment elevation myocardial infarction: analysis from PL-ACS Registry. Kardiol Pol 2010;68:1211-1217.

23. Aldrovandi A, Cademartiri F, Arduini D, et al. Computed tomography coronary angiography in patients with acute myocardial infarction without significant coronary stenosis. Circulation 2012;126:3000-3007.

24. Ibanez B, James S, Agewall S, et al. 2017 ESC guidelines for the management of acute myocardial infarction in patients presenting with ST-segment elevation: the task force for the management of acute myocardial infarction in patients 
presenting with ST-segment elevation of the European Society of Cardiology (ESC). Eur Heart J 2018;39:119-177.

25. von Jeinsen B, Tzikas S, Pioro G, et al. Troponin I assay for identification of a significant coronary stenosis in patients with suspected acute myocardial infarction and wide QRS complex. PLoS One 2016;11:e0154724.

26. Sanchis J, Abellan L, Garcia-Blas S, et al. Usefulness of delta troponin for diagnosis and prognosis assessment of nonST-segment elevation acute chest pain. Eur Heart J Acute Cardiovasc Care 2016;5:399-406.

27. Giannitsis E, Kurz K, Hallermayer K, Jarausch J, Jaffe AS, Katus HA. Analytical validation of a high-sensitivity cardiac troponin T assay. Clin Chem 2010;56:254-261. 